\title{
Thermal wave interferometry measurements correlated to microstructural properties and bonding behavior of thermally sprayed coatings
}

\author{
Jannik Arbogast ${ }^{1,2}$, Rolf Schaller ${ }^{1}$, \\ Magnus Rohde ${ }^{2}$ and Hans Jürgen Seifert ${ }^{2}$ \\ Jannik Arbogast \\ Mercedes-Benz AG, Mercedesstraße 140, 70327 Stuttgart, Germany \\ jannik.arbogast@daimler.com
}

\begin{abstract}
In order to guarantee the life-long functionality of modern combustion engines, the bond strength of thermally sprayed cylinder coatings is one crucial property monitored during the large-scale production of modern power trains. However, since the bond strength between cylinder coating and crankcase substrate can only be measured by destructive off-site testing, a fast and reliable non-destructive method characterizing the bonding behavior of cylinder coatings is highly desired. As the presence of defects in the microstructure lowers the bond strength of cylinder coatings, assessing the defect morphology by non-destructive thermal diffusivity measurements shows promising correlation between thermal and mechanical behavior. Laser-excited lock-in thermography is used to apply thermal wave interferometry (TWI) measurements on wire arc sprayed cylinder coatings of passenger car engines. Measured thermal diffusivity values of these coatings show significant variations along the investigated liners. Further, destructive bonding testing as well as microstructural analysis of the investigated cylinder coatings are acquired to evaluate mechanical and microstructural properties. Investigation of the relationship between thermal diffusivity, bonding behavior and microstructure disclose major correlations between the observed quantities. The application of thermal diffusivity measurements as a nondestructive testing method to evaluate the bond strength of thermally sprayed cylinder coatings is discussed in this work.
\end{abstract}

Keywords: thermal wave interferometry, thermal diffusivity measurements, thermally sprayed coatings, bond testing, cross section microscopy, image analysis

\section{Introduction}

Improving the efficiency of modern power trains and thus reducing $\mathrm{CO}_{2}$ emissions, thermally sprayed cylinder coatings replaced cast iron liners over the last decades [1. Low-alloyed carbon steel coatings are applied using wire arc spraying on mechanically activated cast aluminum-alloy crankcases. Following mechanical treatments including surfacefinishing through honing create mirror-like cylinder liners only a few hundreds of micrometer thick. Thermally sprayed cylinder coatings have proven to lower the size and weight of a combustion engine crankcase and further to cut down the frictional losses between pistons and cylinder running surface, thus reducing the fuel consumption of a modern combustion engine by approximately $3 \%$ [1, 2]. However, to guarantee durability and life-long functionality of the combustion engine, mechanical properties such as the bond strength of the cylinder coating to the substrate have to be monitored during large-scale production. By now, bond strength can only be evaluated by destructive off-site testing. During pull-off adhesion testing (PAT ${ }^{\mathrm{TM}}$ ), elements are glued on the running surface and pulled off hydraulically removing the coating from the substrate. By measuring the applied maximum force, the bond strength can be calculated. As a matter of destructive testing, investigated crankcases cannot be lead back into the production process but need to be recycled. Hence, fast and reliable non-destructive testing of the bond strength is highly desired to further increase the efficiency and to lower the costs of the production process. In order to apply an alternative testing method, coating characteristics influencing the bonding behavior have to be known. As shown in the publication by Schilder et al. 3], the microstructure of thermally sprayed cylinder coatings directly influences the coating bonding behavior. Binding errors, pores and splat interfaces define decisive defects of the material influencing its mechanical properties due to their amount, size and shape [4, 5, 6, 7. Lamellar defects lower the real contact area between overlapping splats within a thermally sprayed coating hence weakening its vertical bond strength [8, 9, 10, 11]. Therefore,

\footnotetext{
${ }^{1}$ Mercedes-Benz AG, Stuttgart, Germany

${ }^{2}$ Karlsruhe Institute of Technology, Karlsruhe, Germany
} 
assessing the defect structure of thermally sprayed cylinder coatings using non-destructive thermal diffusivity measurements 12, 13, 14, 15, 16, one may be able to characterize the bonding behavior without destructive pull-off testing.

Recently, our group has published the investigation of thermal diffusivity measurements and microstructural analysis of cylinder bore coatings to reveal direct influences of the described coating defects on the thermal characteristics [17. The presence of laterally expanded splat interfaces in the coating microstructure lower the resulting thermal diffusivity by up to $50 \%$. As thermal diffusivity measurements being truly sensitive to the occurrence of coating defects, this contribution focuses on the interaction of thermal diffusivity, microstructure and bond strength of wire arc sprayed cylinder coatings. The main objective of this work is to characterize the bonding behavior of thermally sprayed cylinder coatings non-destructively. In this context, laser-excited lock-in thermography is used applying TWI measurements to investigate the thermal diffusivity of thermally sprayed cylinder coatings nondestructively. As shown in [3, a varying microstructure depending on the liner position is present. Therefore, it is expected that the thermal diffusivity values do also vary along the liner position due to the underlying microstructure. Hence, cross section specimens will be cut out off the crankcases to access and evaluate the coatings' microstructures via optical microscopy. These cross section images will be analyzed quantitatively by image analysis algorithms to process the coating defects and correlate them to the measured thermal diffusivity values. Additionally, destructive bond testing will determine the coating bonding behavior and will be compared to the observed coating microstructure and thermal diffusivity values. Whereas laterally splat interfaces are known to reduce the bond strength of the cylinder coatings, also thermal diffusivity values are expected to be lowered at the respective positions within the crankcase due to the underlying microstructure. A strong correlation between the bonding behavior and the thermal characteristics of the coatings should be observable. Additional methods such as the analysis of the fracture surface after bond testing will be applied to obtain a holistic consideration of the relation between coating microstructure and thermal as well as mechanical characteristics.
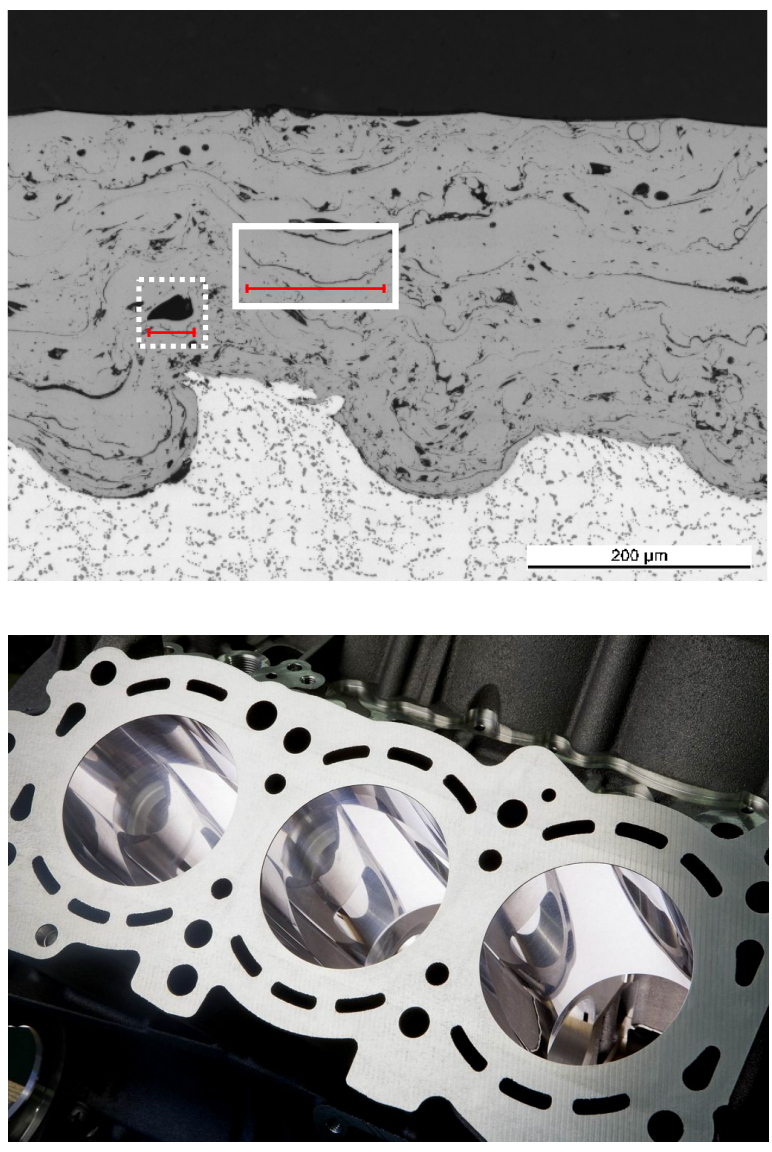

Figure 1: Top: Optical microscopy image of a cross section from a cylinder coating. The functional coating is shown in dark grey, while the aluminum substrate is light grey. The substrate shows the mechanical roughening profile, which leads to sustainable clamping of the coating to the substrate. Further, the cross section image shows the typical morphology of thermally sprayed coatings, while a lateral splat interface (solid box) and a spherical pore (dotted box) are highlighted. The red line indicates the horizontal elongation of the highlighted defects. Bottom: Photography of a typical crankcase including the mirror-like cylinder coating.

\section{Materials - Wire arc sprayed cylinder coatings}

The large-scale production process of wire arc sprayed cylinder coatings may be divided into mechanical pretreatments of the cast crankcases, thermal spraying itself and into surfacefinishing steps through honing. First, mechanical roughening of the cylinder bores within cast aluminum alloy crankcases is required to create a wave-shaped roughening profile with defined as well as non-defined macro- and microstructure (Fig. 1). The roughened substrate profile is crucial to achieve sustainable clamping of the coating to the substrate and shows vertical extensions of up to $150 \mu \mathrm{m}$ [1. Further, neighboring peaks of the roughened substrate exhibit horizontal distances of about $200-300 \mu \mathrm{m}$. Afterwards, crankcases are heated in an oven before thermal spraying to preheat the surfaces. Preheated substrates are favorable for the occurrence of diffusive bond mechanisms between coating and substrate thus increasing the overall bond strength 8,18 . Also, oily residuals of the interface may evaporate during the heating process, since contamination on the surface of the activated substrate lower the bond between coating and substrate [18.

A rotating torch is plunged into the cylinder bores to coat the roughened substrate for all cylinders consecutively. Two wires of low-alloyed carbon steel are carried into the torch, as an electrical voltage applied to the wires causes an arc and melts the coating material at the tip of the torch [19]. Process gas forms a spraying jet of the molten droplets accelerating them towards the substrate, while the torch rotates and moves within the crankcase [19. Spray jet geometry, torch movement during spraying as well as the roughening profile are optimized to guarantee highest possible back-filling of the roughening structure. Avoiding globular pores and unfilled volume within the coating, the bond strength of coating to substrate is enhanced [18]. Further, the used process gas forms a protective atmosphere to reduce oxidation of the coating 
material during the spray process. The molten particles hit the substrate and cool down consecutively, which builds up a typical lamellar structure of partially separated particles and pores. The coating exhibits several different defects due to the coating formation, while two representative defect types are highlighted in Fig. 1. This morphology defines both thermal and mechanical properties and is crucial for all following investigations [4, 6, 20, 21, 22,

However, computational fluid dynamic (CFD) simulations of the complex gas flow during thermal spraying within a cylinder bore reveal that the oxygen mass fraction in the atmosphere surrounding the spraying jet strongly influence the lamellar structure of the coating [3]. Higher mass fraction of oxygen during the coating process leads to enhanced in-flight oxidation of the spraying droplets hence increasing the amount of laterally orientated splat interfaces within the coating microstructure. Eliminating oxygen almost entirely during the coating formation, rather dense structures with few horizontal voids occur [3, 4, 23. Further, excessive bond testing revealed strong influence of the coating's microstructure on its respective bonding behavior. In the presence of many lamellar voids, coating bond decreases dramatically. Thus, lower amount of horizontal splat interfaces favor high bond strengths of thermally sprayed cylinder coatings 3 .

The production process chain is completed by multiple machining steps including surface-finishing through honing, which create the required mirror-like surface structure of the cylinder coatings for latter operation in a combustion engine (Fig. 1). The final coatings exhibit material thicknesses of a few $100 \mu \mathrm{m}$.

\section{Methods}

Measurements and samples for all following methods are taken at positions $10 \mathrm{~mm}, 40 \mathrm{~mm}, 70 \mathrm{~mm}, 100 \mathrm{~mm}$ and $130 \mathrm{~mm}$ below the cylinder head sealing surface for all cylinder liners at four different angle positions. The described measurements and analysis are performed for three different crankcases of type OM656 (Mercedes-Benz AG, Stuttgart, Germany), which were selected randomly from the large-scale production. Overall, 360 data points combining thermal diffusivity measurements and destructive bond testing are acquired.

\subsection{Thermal wave interferometry (TWI)}

Characteristics such as the thermal diffusivity $\alpha$ of thermally sprayed coatings can be measured by thermal wave interferometry (TWI) 17, 22, 24, 25. TWI has been established for a wide range of characterization and testing applications during the past decades $26,27,28,29$. Thermal wave interferometry uses a periodically amplitude-modulated heat source with frequency $f$ to heat the surface of a specimen and hence generates so-called "thermal waves" within the coating material. These waves can be used to investigate thermal and mechanical properties of various kinds of materials non-destructively and contactless [30, 31, 32, 33.

Thermal waves describe strongly damped heat flow based on Fourier's heat conduction equation [34,

$$
\frac{\partial T}{\partial t}=\alpha \nabla^{2} T
$$

propagating diffusively through a material with a characteristic thermal diffusion length $\mu=\sqrt{\alpha / \pi f}$ [35]. The thermal diffusion length describes the penetration depth of a thermal wave where its amplitude is reduced by a factor of $1 / \mathrm{e}$. Based on the principle of heat conduction, thermal waves propagate through the coating material and can be partially reflected at an interface between two connected layers showing a mismatch of their thermal properties. This reflection can be compared to the reflection of magnetic or ultrasonic waves [36], while the reflection coefficient $R_{x, y}$ depends on the thermal effusivities $\epsilon$ of both materials.

$$
R_{x, y}=\frac{\epsilon_{x}-\epsilon_{y}}{\epsilon_{x}+\epsilon_{y}}=\frac{\rho_{x} \cdot c_{x} \cdot \lambda_{x}-\rho_{y} \cdot c_{y} \cdot \lambda_{y}}{\rho_{x} \cdot c_{x} \cdot \lambda_{x}+\rho_{y} \cdot c_{y} \cdot \lambda_{y}}
$$

Here, $\rho$ defines the material density, $c$ is the specific heat capacity and $\lambda$ describes the thermal conductivity, while $x$ and $y$ symbolize the respective layers. In the following, the top layer can be seen as the cylinder coating while the second layer is described by the aluminum-based substrate. Applying continuous thermal waves within the investigated sample, the reflected part of the thermal waves may propagate back to the coating surface and interfere with the incoming ones. This interference leads to a phase-shifted temperature modulation $T(f)$ on the coating surface compared to the incoming heat from the source [35, 37]. Using a one-dimensional approach, the temperature modulation is described by

$$
T(f) \propto \frac{1+R \cdot \exp (-2(1+i) \cdot d / \mu)}{1-R \cdot \exp (-2(1+i) \cdot d / \mu)}
$$

The temperature modulation $T(f)$ is further divided into amplitude and phase, while the phase shift $\phi(f)$ between heat source and surface temperature can be written as

$$
\phi(f)=\tan ^{-1}\left(\frac{-2 R \cdot \exp (-2 \eta \sqrt{f} \cdot \sin (2 \eta \sqrt{f}))}{1-\left(R \cdot \exp (-2 \eta \sqrt{f})^{2}\right)}\right)-\frac{\pi}{4} \quad \text { with } \quad \eta=d \cdot \sqrt{\frac{\pi}{\alpha}}
$$


where $d$ describes the thickness of the coating layer [35. Consequently, if thermal diffusion lengths $\mu$ are chosen to be lower than the respective layer thickness $d$, thermal waves may not reach the interface thus the coating layer can be seen as bulk material. According to Eq. (4), $\eta$ is proportional to both the material thickness and the square root of the inverse thermal diffusivity. Consequently, thermal diffusivity values can only be assessed by TWI measurements, if the material thickness is measured by a reference method.

TWI measurements are performed on a laser-excited lock-in thermography system by edevis GmbH (Stuttgart, Germany) as shown in Fig. 2. The system includes a high power diode laser DSC11 with $938 \mathrm{~nm}$ wavelength and $250 \mathrm{~W}$ maximum power (OsTech e.K., Berlin, Germany). A sinusoidal laser amplitude is modulated by a signal generator and transferred onto the sample surface via an optical fiber. The fiber end is placed behind a lens system controlling the shape and size of the laser spot on the sample. Further, the setup also includes an infrared camera type FLIR X6580 sc (FLIR Systems, Wilsonville, USA) allowing for a maximum frame rate of $600 \mathrm{~Hz}$ with a window size of $360 \times 284$ pixels. Using an InSb-based two-dimensional detector array, the peak of the detector's spectral sensitivity is around $5800 \mathrm{~nm}$ wavelength. The camera is connected with the excitation source via the signal generator, such that the camera frame rate is used as a trigger signal for the signal generator. Therefore, camera read out and excitation source are synchronized to minimize a systematical phase delay between these two components. Further, using a dichroic mirror, a coaxial setup can be achieved, such that the laser beam is placed under an angle of $90^{\circ}$ to the specimen and the camera sits behind the dichroic mirror in line to the measured sample. The applied dichroic mirror uses a dielectric coating to be reflective at a small wavelength band between $850-1000 \mathrm{~nm}$, while being highly transmissive for wavelengths higher than $1200 \mathrm{~nm}$. Due to the geometry of the investigated crankcases, the thermal diffusivity of the cylinder coatings can only be measured nondestructively using a second mirror to deflect the laser beam and the detected radiation by another $90^{\circ}$. Therefore, a gold-plated aluminum mirror is plunged into the crankcase, which can be fully rotated to access all angle positions of the cylinder coating. The crankcases are mount on an attachment sitting on a modulator such that the laser beam can be positioned in all cylinders along the entire running surface. The described setup shows an optical resolution of $0.2 \mathrm{~mm}$ per camera pixel.

Further, thermal diffusivity measurements using TWI are performed with a top-hat shaped laser beam and a spot diameter of $20 \mathrm{~mm}$ on the sample surface. The applied laser power is chosen to $125 \mathrm{~W}$ resulting in a power density of $0.4 \mathrm{~W} / \mathrm{mm}^{2}$. The oscillating laser intensity is set to frequencies at 5, 20, 40, 60, 100, and $150 \mathrm{~Hz}$. Higher excitation frequencies are limited by the hardware properties due to the limited camera's frame rate. Lower excitation frequencies are chosen to be unreasonable, since strong systematic errors occur due to lateral heat diffusion. As the phase shift $\phi(f)$ is derived using a one-dimensional heat diffusion model, the described expression in Eq. (4) is only valid if the thermal diffusion length $\mu$ is much smaller than the beam size [25]. For the described application and the respective investigated samples, thermal diffusion lengths range between about $80 \mu \mathrm{m}$ and $800 \mu \mathrm{m}$, which is comparably small to the excitation area. However, to even further minimize the influence of lateral

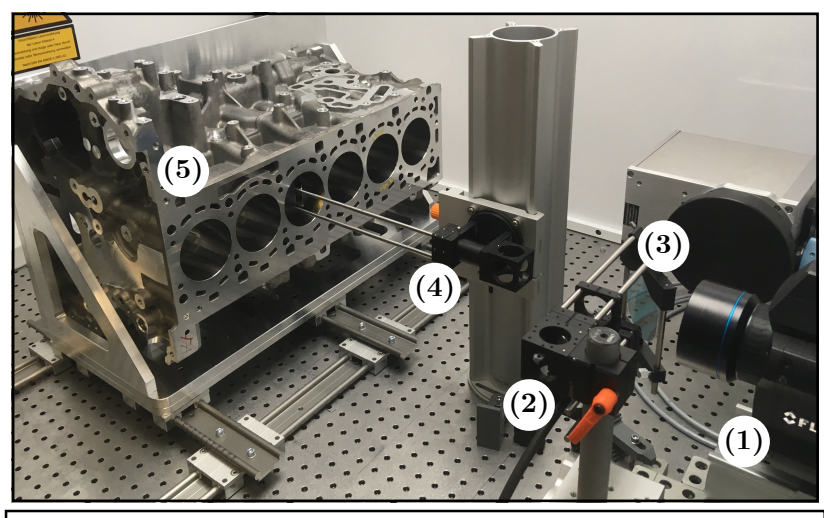

(5) Combustion engine

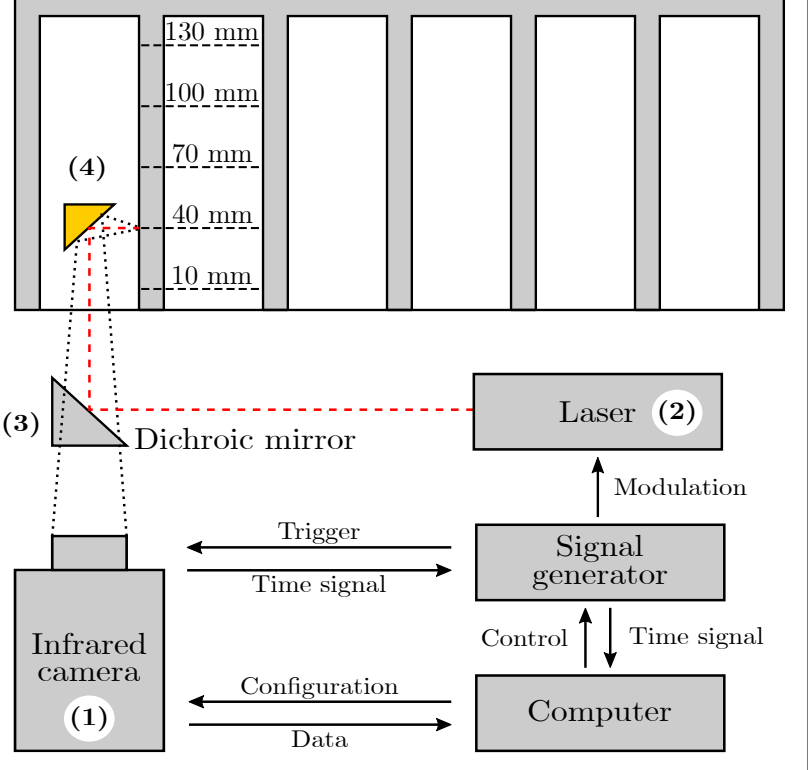

Figure 2: Photography and sketch of the laser-excited lock-in thermography system. (1) Infrared camera, (2) laser fiber end \& lens system, (3) dichroic mirror, (4) gold-plated aluminum mirror including attachment and (5) combustion engine of type OM656 (Mercedes-Benz AG, Stuttgart, Germany). heat flow, temperature values are only evaluated within a spot diameter of $6.7 \mathrm{~mm}$, since lateral heat diffusion dominantly occurs in the outer region of the excited area. Following recommendations by Krankenhagen et al. 38, the sample is preheated for $5 \mathrm{~s}$ in advance of every applied frequency to avoid non-linear sample heating during early excitation. Preliminary experiments have shown that this time scale is sufficient to eliminate non-linear heating effects for all applied modulation frequencies. Afterwards, the infrared camera measures the temperature oscillation on the sample surface for another $5 \mathrm{~s}$, while a Fast Fourier Transformation is applied to calculate its amplitude and phase values. According to Eq. (4), nonlinear least square fitting is used to fit the measured phase information to the model function and hence calculate $\eta$ and $R$ of the coating layer. An offset correction of the 
measured phase values is performed to account for surface influences of the specimens on the measured phase angle as well as systematic errors due to the measurement setup. The one-dimensional theoretical model of thermal waves within a layered structure as found in equation (4) assumes a constant phase shift between the incoming heat flux and the detected surface temperature oscillation of $-\frac{\pi}{4}=-45^{\circ}$ for a thermally thick sample. As the thermal diffusion length $\mu$ decreases with increasing excitation frequency $f$, the model function yields convergence of the measured phase values for a layered sample against $-45^{\circ}$ with increasing excitation frequency. However, deviations of experimental data to the expected convergence value are quite common. Surface influences such as open porosity or grooves from the mechanical treatments are assumed to cause such deviations. Literature often recommends to use a thermally thick reference sample to calibrate the acquired phase values as it is assumed that such a thermally thick reference sample should return a constant phase value of $-45^{\circ}$ 39, 40, 41, 42. However, since a reference sample is not applicable to represent multiple types of surface structures, an alternative approach is used to account for such influences. The model function in equation 4 is expanded by a variable offset $c$ allowing the model function to converge against values different to $-45^{\circ}$. Fitting the measured data using the expanded function with offset correction, the mean coefficient of determination $R^{2}$ increases from 0.978 to 0.999. The mean offset is found at $-45.37^{\circ}$, whereas all offset values range between $-42.06^{\circ}$ and $-47.98^{\circ}$.

Further, by the use of a reference method to measure the material thickness of the layer, $\eta$ allows to calculate the thermal diffusivity value of the coating. Therefore, a magnetic inductive method is applied to measure the coating thicknesses non-destructively [43. The device measures the amount of ferromagnetic material over a paramagnetic substrate. The method is calibrated using X-ray computed tomography data. Due to the roughened substrate, the material thickness of the coating is defined as the mean material volume over the relevant measurement area. Due to the device's comparably large measurement area of $10 \times 10 \mathrm{~mm}^{2}$ as well as the measured phase values on an area showing $6.7 \mathrm{~mm}$ diameter, local lateral variations of the cylinder coating thickness in the scale of $200-300 \mu \mathrm{m}$ will be averaged. Intensive analysis of the reproducibility of the TWI measurements have revealed a standard deviation of the measured thermal diffusivity values of less than $2 \%$. Further, the height difference of the coating due to the local curvature of the cylinder bore is low compared to the depth of field of the camera on the region of interest. Therefore, the authors investigated the effect of a non-flat surface and did not found any significant influences.

\subsection{Pull-off adhesion testing (PAT $\left.T^{T M}\right)$}

The bond strength of thermally sprayed cylinder coatings to the roughened aluminum substrate is measured destructively by in-situ pull-off adhesion testing (PAT ${ }^{\mathrm{TM}}$ ) of type AT101E (DFD Instruments, Kristiansand, Norway). Therefore, test elements with $8 \mathrm{~mm}$ diameter are placed directly on the running surface. The coating is roughened by sandblasting and the test elements are glued onto the surface using a heat-curing one-component adhesive bond based on epoxy resin (Delo Monopox AD 297, DELO Industrie Klebstoffe GmbH \& Co. KGaA, Germany). The test elements are mounted vertically on the running surface by the use of an external adapter board such that the adjacent force is applied perpendicular to the coating structure since misplaced test elements may cause false bond measurements. After curing of the adhesive bond for $120 \mathrm{~min}$ at $180^{\circ}$, the cylinder coating is milled around the testing stamps to preserve a defined measurement area and to remove excessive adhesive material. Finally, the testing stamps are pulled off from the cylinder surface hydraulically causing a break of the coating and the substrate. Measuring the maximum applied force, the bond strength can be calculated due to the defined measurement area according to [44, 45]. The operator inspects the fracture area after the bond strength measurement and divides the observed failure into adhesive or cohesive break. However, in general coating is never fully removed from the substrate thus a composition of adhesive and cohesive failure occurs. Additionally, failure of the adhesive glue may appear as the adhesive is specified to a maximum load of at least 60 MPa. However, multiple experiments have shown that the adhesive glue can overcome loads of 70 MPa. As mostly the specification of the glue is sufficient for bond test, failure of the adhesive glue is noted separately in the testing protocol. The accuracy of the testing device is specified to $\pm 2 \%$ by the manufacturer.

Additionally, fracture after the bond testing is analyzed quantitatively regarding remaining coating material on the aluminum substrate. Failure of the coating after bond testing can be divided into adhesive failure (break between coating and substrate at the interface) and cohesive failure (break within the coating material). Since the coating is never fully removed from the substrate but remain partially bond to the substrate, evaluating the remaining coating material quantitatively can reveal additional information about the interaction of coating and substrate and the respective failure mechanism. Therefore, scanning electron microscopy (SEM) images are taken to measure the amount of remaining coating material on the substrate. SEM images are analyzed using a segmentation algorithm based on a global threshold to separate coating material from substrate. The resulting binarized images only contain remaining coating material thus being summed and normalized to the respective area. The resulting fraction of remaining coating material to substrate reveals the amount of cohesive and adhesive failure during PAT ${ }^{\mathrm{TM}}$ testing. While PAT ${ }^{\mathrm{TM}}$ measurements are performed at the same spots as TWI measurements for all crankcases, four cylinder liners are selected randomly and cut out off the crankcases to evaluate the fracture after PAT $^{T M}$ testing. 
Table 1: ANOVA results of thermal diffusivity and bond strength measurements.

\begin{tabular}{|l|l|ccccc|}
\hline & Category & SS & DF & F & P & Contribution ratio [\%] \\
\hline \hline Therm. Diff. & Crankcase & 12.32 & 2 & 28.00 & $5.38 \mathrm{e}-12$ & 1.18 \\
& Cylinder & 15.39 & 5 & 14.00 & $1.81 \mathrm{e}-12$ & 1.47 \\
& Angle & 100.26 & 3 & 151.92 & $9.32 \mathrm{e}-63$ & 9.60 \\
& Position & 840.24 & 4 & 954.88 & $4.00 \mathrm{e}-185$ & 80.47 \\
& Residuals & 75.90 & 345 & & & 7.27 \\
\hline \hline \multirow{3}{*}{ PAT } & Crankcase & 1255.4 & 2 & 19.87 & $6.82 \mathrm{e}-9$ & 2.89 \\
& Cylinder & 2419.2 & 5 & 15.31 & $1.34 \mathrm{e}-13$ & 5.57 \\
& Angle & 200.4 & 3 & 2.11 & $9.81 \mathrm{e}-2$ & 0.46 \\
& Position & 28677.5 & 4 & 226.91 & $3.15 \mathrm{e}-95$ & 66.00 \\
& Residuals & 10900.5 & 345 & & & 25.09 \\
\hline
\end{tabular}

\subsection{Microstructural analysis}

Light microscopy images of cross sections are used to achieve microstructural analysis of the observed thermally sprayed coatings. Multiple polished cross section specimens are cut out vertically along the cylinder liner with $20 \mathrm{~mm}$ length at the described liner positions. As a single microscopy image covers a field of $650 \times 650 \mu \mathrm{m}$, the entire cross section specimen is covered by about 30 individual microscopy images per measurement position. Quantitative image analysis of the cross section images is carried out using an automated segmentation algorithm based on OpenCV implemented in Python. The applied algorithm focuses on coating defects and extracts all necessary information to quantify the resulting morphology. Global threshold segmentation is used to binarize the cross section images, followed by connected-component-labeling to identify all defects within the coating [46. Latter allows to assess a variety of features of the segmented impurities such as length, orientation and circumference. However, only defects with an area greater than $500 \mathrm{px}$ are processed to reduce calculation time. The analysis algorithm calculates and sums the horizontal elongation of every detected defect (Fig. 1 Top, red line) within one cross section image. The global horizontal defect elongation is normalized to the respective coating area of the coating. This "defect factor" represents the influence of defects and impurities in the coating microstructure orthogonally to the heat conduction of the thermal waves as well as orthogonally to the applied force of the PAT ${ }^{\mathrm{TM}}$ measurements [3, 4, 8. Since the cross section specimens cover about $75 \%$ of the cylinder liner, the image analysis allows to calculate the defect factor over almost the entire liner by appending the calculated defect factors of the individual cross section images regarding to their original position.

\section{Results}

Thermal diffusivity measurements and destructive bond testings are performed for three crankcases within all six cylinders at four angle and five liner positions. Overall, 360 data pairs of thermal diffusivity and bond strength are acquired. The measured data is visualized in Fig. 3 using box plots, as the data is separated by the different categories crankcase, cylinder bore, angle position and liner position. Additionally, an analysis of variance (ANOVA) is conducted in Tab. 1 . to statistically evaluate major influences of the introduced categories on thermal and mechanical coating properties [47]. The value $\mathrm{P}$ indicates the probability of error that the investigated category has a statistical significant influence on the respective coating characteristic. In this context, all $\mathrm{P}$ values are close to zero revealing that all introduced categories have a significant influence on the investigated thermal diffusivity values. However, lowest $\mathrm{P}$ value of the ANOVA according to the thermal diffusivty is found for the liner position. Also highest contribution of the investigated variances is observed with respect to the liner position as the contribution ratio is found at $80.47 \%$ based on the calculated sum of squares (SS). In this this context, strongest and most significant variations of thermal diffusivity can be found with respect to the liner position. Comparing measurement position $10 \mathrm{~mm}$ to $100 \mathrm{~mm}$ in Fig. 3, the median of the thermal diffusivity more than doubles from $4.1 \mathrm{~mm}^{2} / \mathrm{s}$ to $8.7 \mathrm{~mm}^{2} / \mathrm{s}$. The thermal diffusivity shows a strong increase from the cylinder head sealing surface towards $100 \mathrm{~mm}$. However, this increase is followed by a decrease towards position $130 \mathrm{~mm}$ with a respective median thermal diffusivity of $5.6 \mathrm{~mm}^{2} / \mathrm{s}$. The median values and boxes in Fig. 3 clearly indicate two separated levels of the thermal characteristics comparing positions $10 \mathrm{~mm}$ and $40 \mathrm{~mm}$ to position $100 \mathrm{~mm}$ as the whiskers at the respective positions do not overlap. Thermal diffusivity values at positions $70 \mathrm{~mm}$ and $130 \mathrm{~mm}$ are found to lie between these two levels.

Further, according to the ANOVA shown in Tab. 1, all four categories also reveal statistically significant influence on the bond strength. Again, lowest $\mathrm{P}$ value is shown for the liner position, whereas the contribution ratio is found to be at about $66 \%$. Here, a significant part of the observation cannot be traced back to the introduced categories, as the residuals show a contribution ratio of about $25 \%$. However, again the liner position reveals the major statistical influence 

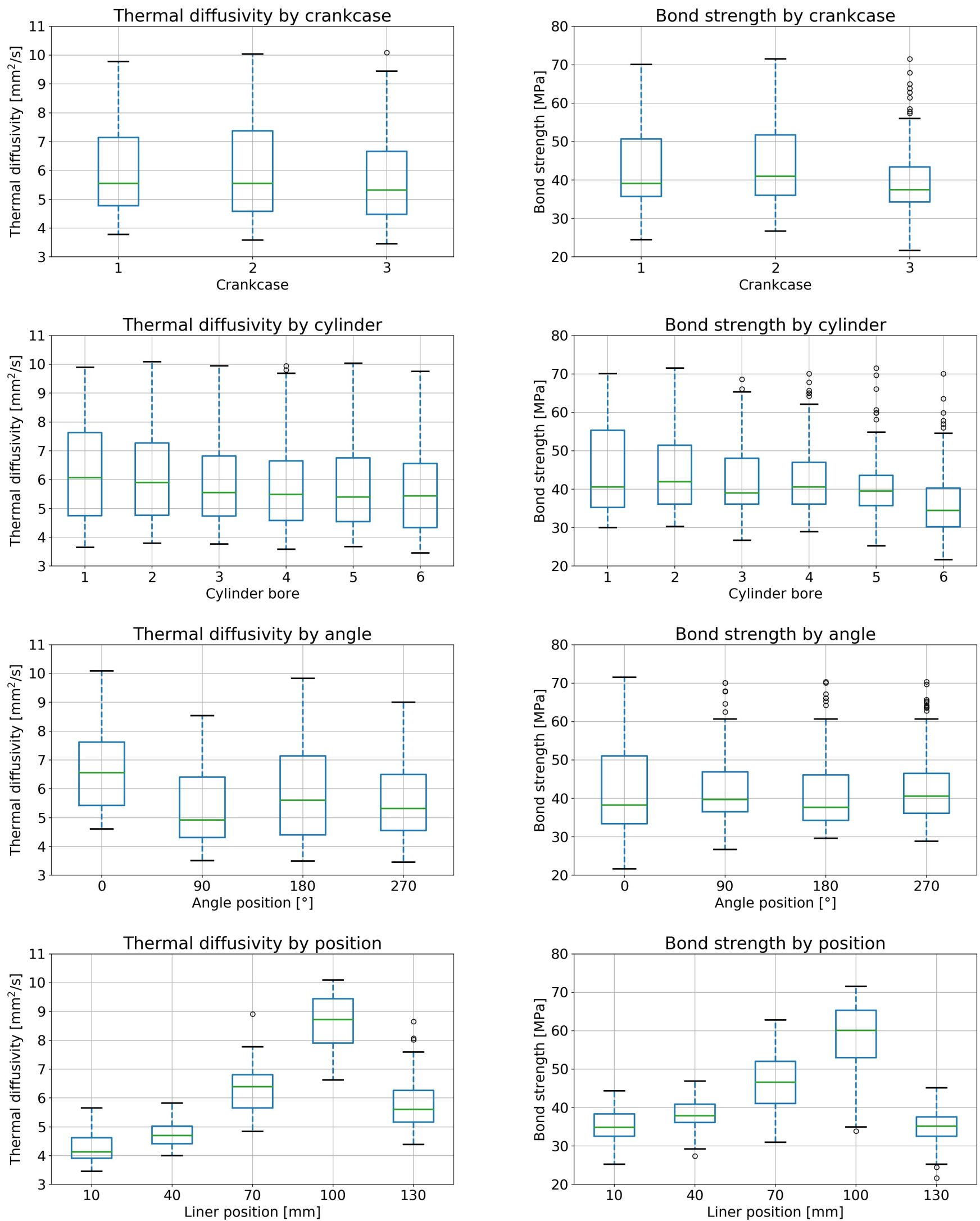

Figure 3: TWI thermal diffusivity measurements (left) and destructive bond strength measurements (right) of the cylinder coatings with respect to the crankcases, cylinders, angle positions and liner positions. The bond strength is normed to the minimum required boding strength in large-scale production. All shown plots include 360 individual data points. Boxplots: The green lines represents the median of the underlying data; boxes indicate the upper/lower quartile; the whiskers are set to a maximum of $1.5 \times$ the interquartile range. Circles mark data outside the whiskers. 
on the measured bond strength. In this context, measured PAT ${ }^{\mathrm{TM}}$ values also vary strongly along the cylinder liner, while the median values of the bond strength show a similar behavior as the thermal diffusivity values. Comparing the obtained medians at positions $10 \mathrm{~mm}$ and $100 \mathrm{~mm}$, bond strength rises by $72 \%$ from $34.8 \mathrm{MPa}$ to $60 \mathrm{MPa}$. Compared to the thermal diffusivity, PAT $^{\mathrm{TM}}$ measurements do also decline towards position $130 \mathrm{~mm}$ to a comparable level as found for position $10 \mathrm{~mm}$. Especially at positions $70 \mathrm{~mm}$ and $100 \mathrm{~mm}$, the quartiles and whiskers of the bond strength measurements indicate that individual data points show an enhanced variation, as the bond strength at position $100 \mathrm{~mm}$ spreads over almost the entire range of measured values. While the median is truly enhanced showing highest available bond strengths compared to the other liner positions, the lower whisker indicates several data points showing comparable PAT ${ }^{T M}$ values to the rest of the liner.

Concluding the statistical analysis, all investigated categories have a statistically significant influence on both thermal and mechanical influences. However, in both cases lowest $\mathrm{P}$ values and highest contribution ratios are found for the liner position. Therefore, the liner position shows the major statistical influence as well as the highest difference between the maximum and minimum median values of thermal and mechanical coating properties along the categories. In this context, further microstructural analysis will only focus on the liner position.

Microstructural analysis is performed using the described segmentation algorithm to analyze cross section images of the cylinder coatings. Two microscopy images of such cross sections at positions $40 \mathrm{~mm}$ and $100 \mathrm{~mm}$ are shown in Fig. 44A. Both cross sections show coating defects highlighted in red, as major differences in the morphology can already be found qualitatively. Fig. 4 A.1 reveals enhanced amount of horizontally expanded splat interfaces at position $40 \mathrm{~mm}$. The coating morphology shows a typical layered structure of partially separated particles as described in the materials section. Further, pronounced coating defects along the aluminum interface can be found. While Fig. 44.2 shows the cross section of the coating at $100 \mathrm{~mm}$, rather round pores are highlighted. Thin, laterally expanded splat interfaces are missing entirely within this image of the respective cross section. The amount and size of coating defects as well as defects along the interface are reduced comparing position $100 \mathrm{~mm}$ to $40 \mathrm{~mm}$.
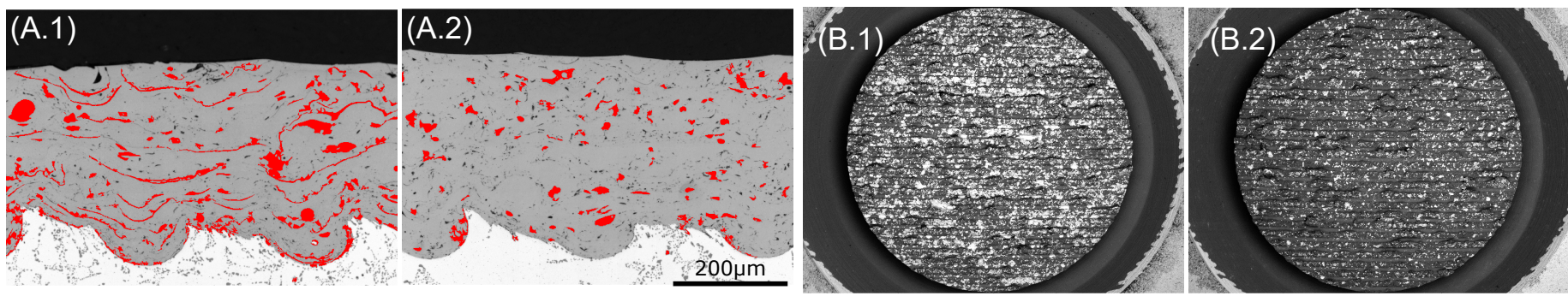

Figure 4: A: Optical microscopy images of the coating cross sections including segmented coating defects highlighted in red (A.1: 40 mm, A.2: $100 \mathrm{~mm}$ ). B: SEM images of the fracture surface after PAT ${ }^{T M}$ (B.1: $40 \mathrm{~mm}$, B.2: $\left.100 \mathrm{~mm}\right)$. The remaining coating material is shown in light grey; aluminum alloy is shown in dark grey.
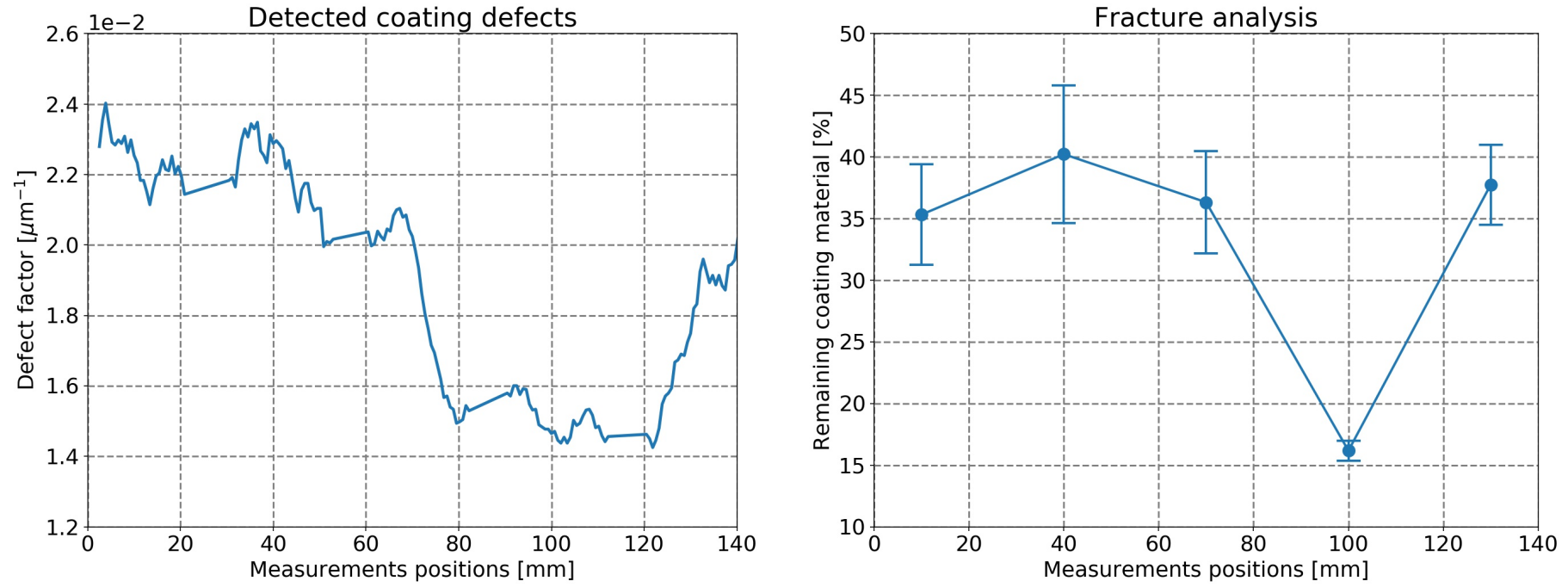

Figure 5: Left: Quantitative analysis of the coating defects using an automated segmentation algorithm. The shown defect factor represents the horizontal elongation of all defects in the microstructure over the substrate. The defect factor is normed on the material thickness of the coating to obtain a unit-less factor. The shown defect factor is calculated for each cross section image individually and assembled to observe the influence of the coating defect along an entire cylinder liner. Right: Quantitative analysis of the acquired SEM images of the fracture after PAT ${ }^{T M}$. The SEM images are analyzed regarding the remaining coating material on the tested area. The whiskers represent the standard deviation of the underlying data. 
As the liner position reveals the strongest influence on both thermal and mechanical properties according to Fig. 3 further quantitative cross section analysis will focus on the influence of the microstructure according to the liner position. Fig. 5 Left illustrates the calculated defect factor along one cylinder liner. The shown values include the quantitative analysis of about 150 individual microscopy images. The calculated defect factor of each image is arranged with respect to its original position along the liner. Liner sectors showing no cross section preparation are interpolated by a linear regression. The defect factor shows a moderate decline starting at $0 \mathrm{~mm}$ towards $70 \mathrm{~mm}$ followed by a strong gradient to position $80 \mathrm{~mm}$. While the defect factor is almost constant between $80 \mathrm{~mm}$ and $120 \mathrm{~mm}$, it increases strongly towards position $140 \mathrm{~mm}$ up to a comparable level as in position $70 \mathrm{~mm}$. Comparing position $40 \mathrm{~mm}$ and $100 \mathrm{~mm}$, two separated levels of the defect morphology can be found along the liner. Here, the defect factor reduces by over $35 \%$ analyzing these two measurement positions.

Additionally, corresponding SEM images of the fracture after PAT ${ }^{T M}$ at positions $40 \mathrm{~mm}$ and $100 \mathrm{~mm}$ are shown in Fig. $4 \mathrm{~B}$. The images represent the fracture of the cylinder liner, while remaining coating material is shown in light grey and aluminum substrate is shown in dark grey. Comparison of the SEM images illustrate two different fractions of remaining coating material to the aluminum substrate. Quantitative analysis of the SEM images using a global threshold to separate coating material and substrate is performed along four different cylinder liners at the described five measurement positions. Again, clearly two separated levels are observed comparing position $100 \mathrm{~mm}$ to the rest of the liner. Fig. 5 Right shows the quantitative analysis of the fracture and reveals a decline of remaining coating material on the substrate after PAT $^{\mathrm{TM}}$ from $40.2 \%$ to $16.2 \%$ for positions $40 \mathrm{~mm}$ and $100 \mathrm{~mm}$, respectively. Further, also in positions $10 \mathrm{~mm}, 70 \mathrm{~mm}$ and $130 \mathrm{~mm}$ increased amount of coating material covers the substrate compared to position $100 \mathrm{~mm}$. Additionally, standard deviation at position $100 \mathrm{~mm}$ is lowered in contrast to the remaining measurement positions.

\section{Discussion}

All shown measurements reveal strong variations of thermal and mechanical layer properties essentially along the cylinder liner. Since this contribution aims to combine thermal and mechanical properties of thermally sprayed coatings, the observed correlations are connected by the varying coating microstructure. As Schilder et al. presented in their work [3, the occurrence of defects and impurities in the microstructure of thermally sprayed cylinder coatings depends on the amount of oxygen during twin wire arc spraying. Computational fluid dynamic simulations of the complex gas flow during the coating process as well as coating experiments have shown that the mass fraction of oxygen in the atmosphere surrounding the spray jet strongly varies along the cylinder liner during the coating process. This observed varying oxygen content results in a varying coating microstructure and enhanced amount of defects and impurities depending on the position in the crankcase. High oxygen content found in the upper parts as well as in the lowest part near the crankcase shaft at position $>120 \mathrm{~mm}$ of the cylinder bores leads to enhanced oxidation of the spray particles during the coating process resulting in distinct laterally orientated splat interfaces and the observed morphology. A coating process in absence of oxygen results in dense microstructures with very few defects and can be found especially in the region

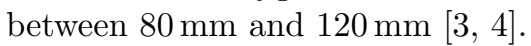

Since various publications have proven the influence of such coating defects on the thermal as well as mechanical properties 3, 5, 17, 21, 48, the observed variations of thermal diffusivity as well as coating bond along the liner can be traced back to the varying defect morphology. Therefore, quantitative analysis of the cross section images focuses on the horizontal elongation of the observed defects by calculating the introduced defect factor allowing to connect the influence of coating defects with its thermal and mechanical properties. Laterally expanded splat interfaces appear to be dominant at influencing the vertical heat conduction, while such defects build up multiple thermal barriers lowering the thermal diffusivity from surface to substrate 8,49 . TWI measurements can be seen as integral measurements of the total influence of the microstructure defects on the thermal behavior of the coatings. The absence of horizontal defects represented by a lowered defect factor at measurement position $100 \mathrm{~mm}$ (Fig. 5 Left) leads to an increase of the thermal diffusivity of over $100 \%$ as seen in Fig. 3 The observed defect factor reveals two clearly separated levels of coating microstructure comparing the upper and lower parts of the liner, causing two separated levels found for the thermal diffusivity at the respective positions $10 \mathrm{~mm}$ and $40 \mathrm{~mm}$ as well as $100 \mathrm{~mm}$. Further, Fig. 5Left shows a strong gradient of the calculated defect factor at positions $70 \mathrm{~mm}$ and $130 \mathrm{~mm}$. Therefore, enhanced variations of TWI measurements are caused by the rapid change of the microstructure within the observed measurement position. Obviously, the thermal diffusivity is affected by the microstructure and there, it is correlated with the defect factor as shown in Fig. 6] Left. Multiple cross section specimens are analyzed regarding their defect distribution. Regarding each data point, the respective thermal diffusivity is shown over the median value of the defect factor. A lowered amount of horizontal defect elongation clearly results in higher thermal diffusivity of the coating and vice versa. However, the shown correlation may reveal a rather quadratic trend than a linear correlation. The shown curve appears to flatten for higher defect factors and respective lowered thermal diffusivity values. Still, the shown data points relate with a correlation factor of -0.844 . Thus, TWI measurements seem to be truly sensitive regarding this microstructural characteristic. 

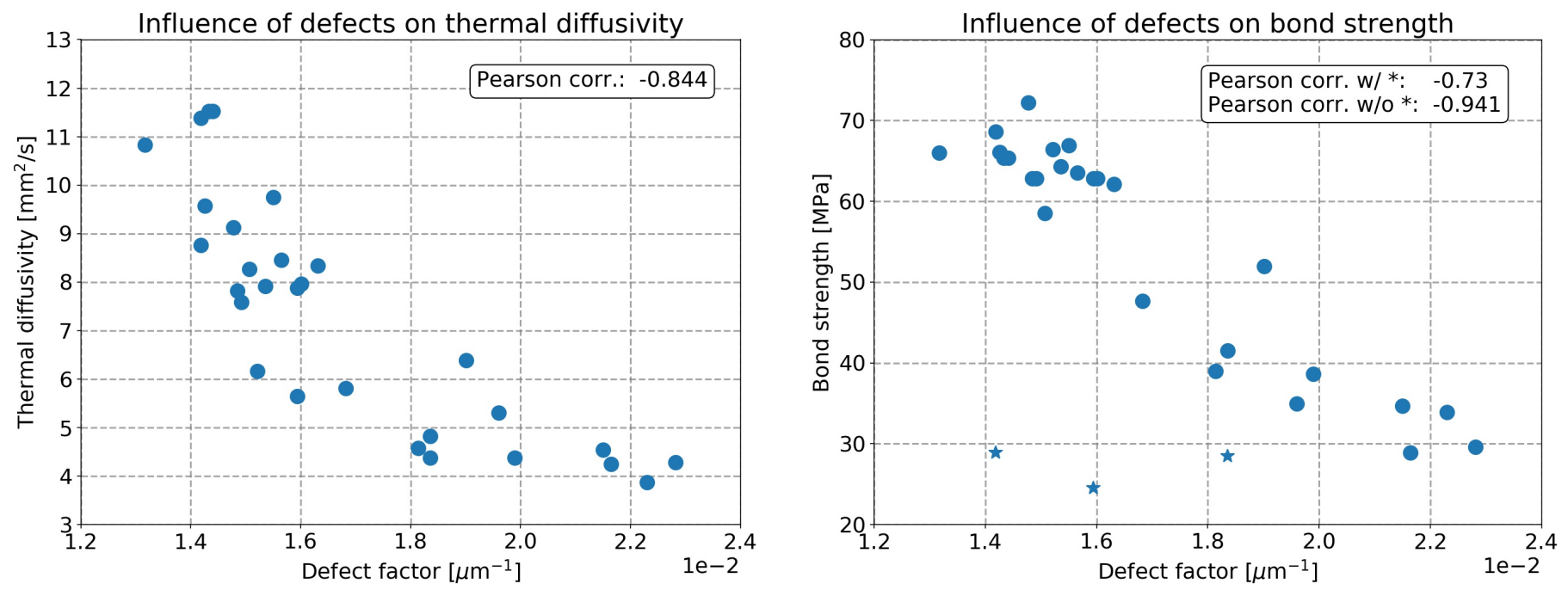

Figure 6: Multiple cross sections are analyzed regarding their defect distribution. The shown data points represent median values of all evaluated microscopy images per cross section. Both thermal diffusivity measurements (left) and pull-off adhesion testing (right) are performed at the conventional measurement positions in advance of cross section preparation. Due to the destructive PAT ${ }^{T M}$ measurements, cross sections are cut out off the liner closest possible to the fracture. Data points found aside from the reciprocal trend of bond strength to defect factor are marked with stars.

Further, microstructure influences on the coating bond strength can also be explained with the calculated defect factor. The bonding behavior of a coating to a substrate need to be separated into the two different mechanisms of adhesive and cohesive bond. The overall coating bond however conjuncts both mechanisms as failure occurs at the weakest part of the system. While coating defects build up thermal barriers, they may also reduce the inner coating bond by reducing the truly bond coating ratio [49. Oxides are known to influence the bond between individual splats, while increased oxide content in a sprayed layer reduces the interlamellar bond of the coating thus reducing the cohesive bond strength [19, 50. Since PAT $^{\text {TM }}$ is applied orthogonally to the substrate orientation, the horizontal defect elongation can be seen as a quantity of the representative interlamellar cohesive bond. Thus, distinct relations between the coating microstructure and the tested bond strength can be found with the applied methods. Horizontal defects cause an overall lowered bond strength of the coating system, while the shown reduction of the defect factor in Fig. 5 Left results in an increase of bond strength of over $72 \%$ at position $100 \mathrm{~mm}$. The strong increase of the defect factor towards $130 \mathrm{~mm}$ forms a decrease of the bond strength to a comparable level as in positions $10 \mathrm{~mm}$ and $40 \mathrm{~mm}$. In general, bonding behavior inversely proportional to the defect factor can be assumed comparing PAT ${ }^{T M}$ measurement in Fig. 3 and Fig. 5 Left. This assumption is supported by showing the acquired PAT ${ }^{T M}$ measurements over the defect factor in Fig. 6 Right. Again, the same cross section specimens as seen in 6 Left are shown over their respective bond strength values. High bonding values are truly the consequence of low defect factors. Condensed data points showing high bond strength and low defect factor support the idea that the absence of laterally expanded splat interfaces increases the overall bond strength dramatically. The observed correlation of the defect factor and bond strength in Fig. 6 does not show any data points combining high bond strength and enhanced defect factor. This supports the assumption that the presence of defects and impurities directly causes a distinct reduction of the bond strength for thermally sprayed cylinder coatings. Highest bond strengths can only be observed if a dense coating can be formed showing no lateral defects lowering the cohesive behavior. However, as the defect factor does only represent the cohesive bond, measurements can be found showing low defect factor and low bond strength. The respective data points are marked by stars in figure 6. Aside from the reciprocal trend, weakened adhesion can cause bond failure even for a coating obtaining almost no defects in its microstructure. Contamination of the substrate interface or defective roughening structures are known to influence the adhesive bond of a coating to a substrate [18. Oily contamination prevent diffusion bond between coating and substrate, which leaves the adhesion mechanism to be only mechanical. While the production is set to protect the interface from contamination before the coating spray process, still the substrate cannot be kept clean and protected entirely. Further, defective roughening structures caused by broken roughening tools can also lower the mechanical clamping of the solidified coating to the substrate interface. While roughening tools are monitored after machining of each individual crankcase, however abrasive wear or break of the used tools can cause variations in the roughening profile thus leading to lowered adhesive bond. Both influences cannot be observed and explained applying the introduced defect factor to the cross section images. Still, statistical analysis of all shown data points in Fig. 6 Right reveal a correlation factor of -0.73 . Even higher statistical significance is observed in the absence of the adhesive failure, while a Pearson correlation of -0.941 between the defect factor and the bond strength can be calculated.

Additionally, quantitative SEM image analysis of the fracture area after $\mathrm{PAT}^{\mathrm{TM}}$ underlines the influence of laterally ex- 
panded defects on the bond strength of the coatings. The quantitative fracture analysis can be seen as an indicator of the proportion of cohesive to adhesive failure during PAT ${ }^{\text {TM }}$. SEM images exhibit an enhanced amount of remaining coating material on the aluminum substrate of up to $40 \%$ at position $40 \mathrm{~mm}$, thus reduced bond values result in enhanced remaining coating material after testing. Here, lateral defects weaken the inner coating bond, increasing failure within the coating resulting in enhanced cohesive break. In contrast, microstructure analysis at measurement position $100 \mathrm{~mm}$ show almost no laterally expanded splat interface as well as lowered amount of remaining coating material on the substrate. This reveals a direct influence of lateral defects on the overall coating bond. If cylinder coatings are manufactured showing no lateral defects as in position $100 \mathrm{~mm}, \mathrm{PAT}^{\mathrm{TM}}$ removes almost the entire coating from the substrate as failure appears at the coating-substrate interface. Therefore, the calculated defect factor appears to represent the main influence of coating failure during bond testing and can be used to characterize the bonding properties of thermally sprayed cylinder bore coatings.

As previously shown, measurements and statistical analysis emphasize a strong correlation between the thermal and mechanical properties of the introduced cylinder coatings based on the underlying microstructure. Therefore, Fig. 7 presents the correlation of all acquired data points of TWI and PAT ${ }^{T M}$ measurements. Statistical analysis of the thermal diffusivity and bond strength measurements reveals a correlation factor of 0.741 of the individual data points. Due to the lamellar coating formation and the presence of laterally orientated splat interfaces, higher bonding strengths require high thermal diffusivity values. While high thermal diffusivity values indicate a coating structure in absence of horizontal voids, the cohesive bond of the coating is enhanced. As a matter of fact, no measurement data can be found showing low thermal diffusivity and high bond strength. The presence of laterally expanded splat interfaces lower the thermal diffusivity and weaken the cohesive bond strength of the coating. Consequently, the correlation between thermal diffusivity and cohesive bond strength founds on the introduced defect factor. However, particular data points can be detected aside from the gen-

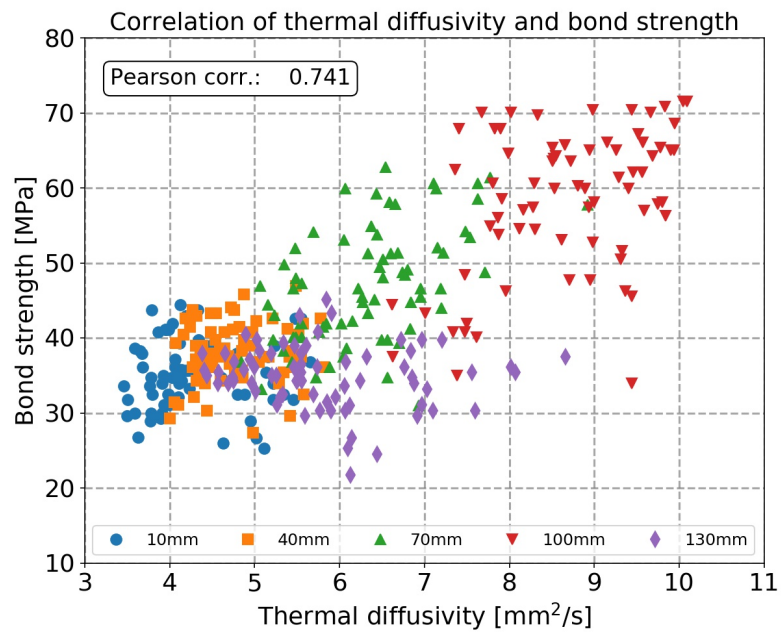

Figure 7: Correlation between thermal diffusivity and bond strength based on the TWI and PAT ${ }^{\mathrm{TM}}$ measurements presented in Fig. 3 The data points are divided by color and shape according to the measurement position along the cylinder liners.

eral trend showing lowered bond strength and high thermal

diffusivity. Here, the adhesion bonding behavior must be reduced while the inner coating strength is not affected by coating defects. Since the presented TWI interferometry is used to determine the integral thermal diffusivity of the coating, singular effects at the coating-substrate-interface cannot be recognized. Therefore, correlation between TWI measurements and $\mathrm{PAT}^{\mathrm{TM}}$ does reveal the relation of thermal diffusivity and cohesive bonding behavior. If external influences such as oily contamination or failure of the roughening profile can be reduced and the resulting adhesive bond strength is kept high, overall bond strength can be directly correlated to TWI measurements.

\section{Conclusion}

Non-destructive thermal diffusivity measurements, destructive bond testing as well as microstructural analysis are used to correlate the thermal diffusivity with microstructural and mechanical properties of thermally sprayed cylinder bore coatings. The measured thermal diffusivity values show strong and systematic variations of over $100 \%$ along the cylinder liner depending on the position in the crankcase. Further, bond measurements reveal that the bond strength shows comparable behavior as the measured thermal diffusivity. Additionally, microstructural analysis of the coating defect morphology as well as analysis the fracture area after bond testing are performed to further explain the observed relation of thermal and mechanical properties of the cylinder coatings. Coating defects and impurities build up multiple thermal barriers orthogonally to the vertical heat conduction, revealing a direct correlation of the measured thermal diffusivity values and the observed coating defect morphology. Further, as coating defects are known to reduce the interlamellar cohesive bond strength of sprayed layers, the observed microstructure has a dominant influence on the overall coating bond, thus a statistical correlation of thermal diffusivity to bond strength values of 0.741 is observed. Whereas laterally orientated splat interfaces lead to enhanced cohesive failure, rather adhesive failure can be found at measurement positions showing almost no such defect types.

Thermal diffusivity and cohesive bond may be directly correlated, whereas the correlation of the individual data points reveal a pronounced relation between thermal and mechanical properties of the investigated coatings. Since the observed varying coating microstructure seem to be the predominant coating characteristic defining the overall bonding behavior of the coatings, TWI opens the ability to analyze and assess the defect distribution in the microstructure of the cylinder 
coating. Being truly contactless and non-destructively, TWI may be applied for automated inline testing of the coating quality as a fast and reliable testing procedure. In this context, TWI defines a useful testing method showing promising results characterizing the bond strength.

However, as weakened adhesion of the coating to the substrate cannot be determined using thermal diffusivity measurements yet, the total bond strength can only be partially assessed using TWI. With the intent to fully replace PAT ${ }^{\mathrm{TM}}$ measurements within the large-scale production, influences reducing the adhesive bond have to be eliminated. However, combining PAT $^{\mathrm{TM}}$ and TWI measurements, one may be able to distinguish between adhesive and cohesive failure without excessive preparation and study of scanning electron microscopy images.

\section{References}

[1] M. Schmidt, H. Spieth, J. Bauer, C. Haubach, Prozesskette NANOSLIDE@, in: 100 Betriebe für RessourceneffizienzBand 1, Springer, 2017, pp. 234-237. doi:10.1007/978-3-662-53367-3.

[2] J. Schommers, H. Scheib, M. Hartweg, A. Bosler, Reibungsminimierung bei Verbrennungsmotoren, MTZMotortechnische Zeitschrift 74 (7-8) (2013) 566-573. doi:https://doi.org/10.1007/s35146-013-0170-y.

[3] B. Schilder, A. Garling, F. Reimer, M. Hamann, R. Joos, J. Hüger, M. Pöhlmann, T. Lampke, Cfd enhanced thermal spray process for coating of cylinder bores of car engines, Journal of Thermal Spray Technology (2020) 1-14doi:https://doi.org/10.1007/s11666-020-01011-y.

[4] S. Deshpande, S. Sampath, H. Zhang, Mechanisms of oxidation and its role in microstructural evolution of metallic thermal spray coatings - case study for ni-al, Surface and Coatings Technology 200 (18-19) (2006) 5395-5406. doi: https://doi.org/10.1016/j.surfcoat.2005.07.072.

[5] L. Pawlowski, P. Fauchais, Thermal transport properties of thermally sprayed coatings, International materials reviews 37 (1) (1992) 271-289. doi:https://doi.org/10.1179/imr.1992.37.1.271.

[6] I. Gedzevicius, A. Valiulis, Analysis of wire arc spraying process variables on coatings properties, Journal of Materials Processing Technology 175 (1-3) (2006) 206-211. doi:https://doi.org/10.1016/j.jmatprotec.2005.04.019

[7] G. M. Smith, M. Resnick, B. Kjellman, J. Wigren, G. Dwivedi, S. Sampath, Orientation-dependent mechanical and thermal properties of plasma-sprayed ceramics, Journal of the American Ceramic Society 101 (6) (2018) 2471-2481. doi:https://doi.org/10.1111/jace.15405

[8] X. Wang, J. Heberlein, E. Pfender, W. Gerberich, Effect of nozzle configuration, gas pressure, and gas type on coating properties in wire arc spray, Journal of Thermal Spray Technology 8 (4) (1999) 565-575. doi:https: //doi.org/10.1361/105996399770350269.

[9] C.-J. Li, G.-J. Yang, Relationships between feedstock structure, particle parameter, coating deposition, microstructure and properties for thermally sprayed conventional and nanostructured wc-co, International Journal of Refractory Metals and Hard Materials 39 (2013) 2-17. doi:https://doi.org/10.1016/j.ijrmhm.2012.03.014.

[10] H. Liao, B. Normand, C. Coddet, Influence of coating microstructure on the abrasive wear resistance of wc/co cermet coatings, Surface and Coatings Technology 124 (2-3) (2000) 235-242. doi:https://doi.org/10.1016/ S0257-8972(99) 00653-2

[11] C. Berndt, R. McPherson, The adhesion of plasma sprayed ceramic coatings to metals, in: Surfaces and Interfaces in Ceramic and Ceramic-Metal Systems, Springer, 1981, pp. 619-628. doi:https://doi.org/10.1007/ 978-1-4684-3947-2_54.

[12] K. Bobzin, T. Brögelmann, C. Kalscheuer, B. Yildirim, M. Welters, Correlation of thermal characteristics and microstructure of multilayer electron beam physical vapor deposition thermal barrier coatings, Thin Solid Films (2020) 138081doi:https://doi.org/10.1016/j.tsf.2020.138081

[13] R. Yang, Y. He, Optically and non-optically excited thermography for composites: A review, Infrared Physics \& Technology 75 (2016) 26-50. doi:https://doi.org/10.1016/j.infrared.2015.12.026.

[14] F. Cernuschi, P. Bison, A. Moscatelli, Microstructural characterization of porous thermal barrier coatings by laser flash technique, Acta Materialia 57 (12) (2009) 3460-3471. doi:https://doi.org/10.1016/j.actamat.2009.03. 041. 
[15] F. Cernuschi, I. Golosnoy, P. Bison, A. Moscatelli, R. Vassen, H.-P. Bossmann, S. Capelli, Microstructural characterization of porous thermal barrier coatings by ir gas porosimetry and sintering forecasts, Acta materialia 61 (1) (2013) 248-262. doi:https://doi.org/10.1016/j.actamat.2012.09.055.

[16] W. Chi, S. Sampath, H. Wang, Microstructure-thermal conductivity relationships for plasma-sprayed yttria-stabilized zirconia coatings, Journal of the American Ceramic Society 91 (8) (2008) 2636-2645. doi:https://doi.org/10. $1111 / \mathrm{j} .1551-2916.2008 .02476 . \mathrm{x}$

[17] J. Arbogast, R. Schaller, S. Lindemann, B. Schilder, M. Rohde, H. J. Seifert, Thermal wave interferometry measurements and microstructural analysis of twin wire arc spray cylinder coatings for passenger car engines, Infrared Physics \& Technology 105 (2020) 103216. doi:https://doi.org/10.1016/j.infrared.2020.103216.

[18] P. L. Fauchais, J. V. Heberlein, M. I. Boulos, Coating characterizations, in: Thermal Spray Fundamentals, Springer, 2014, pp. 1113-1250. doi:https://doi.org/10.1007/978-0-387-68991-3.

[19] J. König, M. Lahres, O. Methner, Quality designed twin wire arc spraying of aluminum bores, Journal of Thermal Spray Technology 24 (1-2) (2015) 63-74. doi:https://doi.org/10.1007/s11666-014-0170-6.

[20] F. Cernuschi, S. Ahmaniemi, P. Vuoristo, T. Mäntylä, Modelling of thermal conductivity of porous materials: application to thick thermal barrier coatings, Journal of the European Ceramic Society 24 (9) (2004) 2657-2667. doi:https://doi.org/10.1016/j.jeurceramsoc.2003.09.012.

[21] Z. Wang, A. Kulkarni, S. Deshpande, T. Nakamura, H. Herman, Effects of pores and interfaces on effective properties of plasma sprayed zirconia coatings, Acta Materialia 51 (18) (2003) 5319-5334. doi:https://doi.org/10.1016/ S1359-6454(03) 00390-2.

[22] P. Patel, D. Almond, Thermal wave testing of plasma-sprayed coatings and a comparison of the effects of coating microstructure on the propagation of thermal and ultrasonic waves, Journal of Materials Science 20 (3) (1985) 955966. doi:https://doi.org/10.1007/BF00585740.

[23] P. Fauchais, A. Vardelle, B. Dussoubs, Quo vadis thermal spraying?, Journal of Thermal Spray Technology 10 (1) (2001) 44-66. doi:https://doi.org/10.1361/105996301770349510.

[24] A. Bento, D. Almond, The accuracy of thermal wave interferometry for the evaluation of thermophysical properties of plasma-sprayed coatings, Measurement Science and Technology 6 (7) (1995) 1022. doi:https://doi.org/10. $1088 / 0957-0233 / 6 / 7 / 025$.

[25] A. Bendada, M. Lamontagne, H. Roberge, Influence of thermal properties on the sensitivity of thermal wave interferometry for the characterization of plasma-sprayed coatings, International Journal of Thermophysics 24 (1) (2003) 207-222. doi:https://doi.org/10.1023/A:1022322517684

[26] O. Breitenstein, W. Warta, M. C. Schubert, Lock-in thermography: Basics and use for evaluating electronic devices and materials, Vol. 10, Springer, 2019. doi:10.1007/978-3-642-02417-7.

[27] G. Busse, D. Wu, W. Karpen, Thermal wave imaging with phase sensitive modulated thermography, Journal of Applied Physics 71 (8) (1992) 3962-3965. doi:https://doi.org/10.1063/1.351366.

[28] X. Maldague, Theory and practice of infrared technology for nondestructive testing, Wiley series in microwave and optical engineering, John Wiley \& Sons, Inc., New York, USA, 2001.

[29] D. Wu, G. Busse, Lock-in thermography for nondestructive evaluation of materials, Revue générale de thermique 37 (8) (1998) 693-703. doi:https://doi.org/10.1016/S0035-3159(98)80047-0.

[30] J. Opsal, A. Rosencwaig, D. L. Willenborg, Thermal-wave detection and thin-film thickness measurements with laser beam deflection, Applied Optics 22 (20) (1983) 3169-3176. doi:https://doi.org/10.1364/A0.22.003169.

[31] A. Salazar, A. Sánchez-Lavega, Thermal diffusivity measurements using linear relations from photothermal wave experiments, Review of scientific instruments 65 (9) (1994) 2896-2900. doi:https://doi.org/10.1063/1.1144635

[32] W. Karpen, D. Wu, G. Busse, Invited article a theoretical model for the measurement of fiber orientation with thermal waves, Research in nondestructive evaluation 11 (4) (1999) 179-197. doi:https://doi.org/10.1007/PL00003920.

[33] F. Cernuschi, P. Bison, A. Figari, S. Marinetti, E. Grinzato, Thermal diffusivity measurements by photothermal and thermographic techniques, International Journal of Thermophysics 25 (2) (2004) 439-457. doi:https://doi.org/ 10.1023/B:IJOT.0000028480.27206.cb. 
[34] J. B. J. Fourier, Théorie du mouvement de la chaleur dans les corps solides, 1824.

[35] C. Bennett, R. Patty, Thermal wave interferometry: a potential application of the photoacoustic effect, Applied Optics 21 (1) (1982) 49-54. doi:https://doi.org/10.1364/A0.21.000049.

[36] D. Almond, P. Patel, I. Pickup, H. Reiter, An evaluation of the suitability of thermal wave interferometry for the testing of plasma sprayed coatings, NDT International 18 (1) (1985) 17-24. doi:https://doi.org/10.1016/ 0308-9126(85) 90038-0.

[37] A. Rosencwaig, A. Gersho, Theory of the photoacoustic effect with solids, Journal of Applied Physics 47 (1) (1976) 64-69. doi:https://doi.org/10.1063/1.322296

[38] R. Krankenhagen, M. Ziegler, C. Maierhofer, Systematic errors in the evaluation of uncorrected data from thermographic lock-in measurements (2018).

[39] A. Gleiter, C. Spiessberger, G. Busse, Phase angle thermography for depth resolved defect characterization, in: AIP Conference Proceedings, Vol. 1096, American Institute of Physics, 2009, pp. 526-532.

[40] W. Bai, B. Wong, Photothermal models for lock-in thermographic evaluation of plates with finite thickness under convection conditions, Journal of applied physics 89 (6) (2001) 3275-3282.

[41] S. Pickering, D. Almond, Matched excitation energy comparison of the pulse and lock-in thermography nde techniques, Ndt \& E International 41 (7) (2008) 501-509.

[42] C. Wallbrink, S. Wade, R. Jones, The effect of size on the quantitative estimation of defect depth in steel structures using lock-in thermography, Journal of applied physics 101 (10) (2007) 104907.

[43] S. Lindemann, Zerstörungsfreie Charakterisierung thermisch gespritzter Zylinderlaufflächen, Ph.D. thesis, Universität Magdeburg (2019).

[44] Norm. DIN EN 582:1994-01: Thermal spraying; determination of tensile adhesive strength (1994).

[45] Norm. DIN EN ISO 4624:2016-08: Paints and varnishes - pull-off test for adhesion (2016). doi:https://dx.doi. org/10.31030/2360041

[46] C. Ronsen, P. Denjiver, Connected Components in Binary Images: The Detection Problem., John Wiley \& Sons, Inc., New York, USA, 1984.

[47] H. Scheffe, The analysis of variance, Vol. 72, John Wiley \& Sons, 1999.

[48] R. McPherson, A model for the thermal conductivity of plasma-sprayed ceramic coatings, Thin Solid Films 112 (1) (1984) 89-95. doi:https://doi.org/10.1016/0040-6090(84)90506-6.

[49] S. Boire-Lavigne, C. Moreau, R. Saint-Jacques, The relationship between the microstructure and thermal diffusivity of plasma-sprayed tungsten coatings, Journal of Thermal Spray Technology 4 (3) (1995) 261-267. doi:https: //doi.org/10.1007/BF02646969.

[50] G. Jandin, H. Liao, Z. Feng, C. Coddet, Correlations between operating conditions, microstructure and mechanical properties of twin wire arc sprayed steel coatings, Materials Science and Engineering: A 349 (1-2) (2003) 298-305. doi:https://doi.org/10.1016/S0921-5093(02)00767-0 